\title{
Inhibition of colon carcinogenesis by post-initiation induction of NQO1 in Sprague-Dawley rats
}

\author{
ASHER BEGLEITER $^{1,2}$, KOSALA SIVANANTHAN ${ }^{1}$, GEORGIA M. LEFAS $^{1}$, \\ ANDREW W. MAKSYMIUK ${ }^{2}$ and RANJANA P. BIRD ${ }^{3}$
}

\author{
${ }^{1}$ Manitoba Institute of Cell Biology, CancerCare Manitoba, Winnipeg; ${ }^{2}$ Department of Internal Medicine, University of \\ Manitoba, Winnipeg, R3E 0V9; ${ }^{3}$ Department of Biological Sciences, University of Windsor, Windsor N9B 3P4, Canada
}

Received December 31, 2008; Accepted February 11, 2009

DOI: 10.3892/or_00000388

\begin{abstract}
Inducers of phase II detoxifying enzymes have been studied as chemopreventive agents for a variety of cancers. Phase II detoxifying enzymes may play a significant role in preventing carcinogen-induced colon cancer at the initiation and post-initiation stage, but the contribution of NAD $(P)$ $\mathrm{H}$ :quinone oxidoreductase 1 (NQO1) to this effect remains unclear. Using the carcinogen-induced colon cancer SpragueDawley rat model, we previously showed that oltipraz selectively induces NQO1 in the colons of these rats without inducing other phase II detoxifying enzymes. We demonstrated that selective induction of NQO1 in the rat colon prior to treatment with a carcinogen significantly inhibited the formation of aberrant crypt foci (ACF). Using the same rat model, we found that rats fed oltipraz containing diet following treatment with the colon carcinogen, azoxymethane (AOM), had $60 \%$ fewer ACF after 12 weeks compared with rats fed a control diet. In addition, rats fed oltipraz containing diet after AOM treatment developed $40 \%$ fewer colon adenomas and fewer colon tumors than rats fed a control diet. There was also a $60 \%$ increase in the percentage of apoptotic cells in ACF from oltipraz fed rats compared with ACF from control fed rats. Together, these results suggest that NQO1 can contribute to inhibition of colon carcinogenesis at the postinitiation stage. A possible mechanism for this effect may be that induction of NQO1 increases apoptosis in carcinogen initiated colonic epithelial cells that prevents these cells from progressing to a neoplastic state. Thus, NQO1 may be an important target for chemoprevention of colon cancer.
\end{abstract}

Correspondence to: Dr Asher Begleiter, Manitoba Institute of Cell Biology, 675 McDermot Avenue, Winnipeg, MB R3E 0V9, Canada

E-mail: begleit@cc.umanitoba.ca

Key words: chemoprevention, colon carcinogenesis, NQO1, postinitiation, rat model, apoptosis

\section{Introduction}

Colorectal cancer represents $11 \%$ of new cancers and $10 \%$ of cancer deaths in North America and occurs equally in men and women (1). Most of these cancers are of a sporadic nature and develop from colonic epithelial cells following initiation by carcinogens. Promoters increase replication of the initiated cells in the colon leading to clonal expansion. Progression involves the evolution of these pre-cancerous lesions through a series of well defined stages from dysplastic epithelium, including the development of early lesions called aberrant crypt foci (ACF), to adenomas, malignant carcinomas and metastatic cancer.

$\mathrm{ACF}$ are characterized by their increased size, irregular and dilated luminal opening, thicker epithelial lining and pericryptal zone compared with normal colonic crypts (2). Colon carcinogens have been shown to induce ACF in a dose-related manner, and known inhibitors or promoters of colon carcinogenesis inhibit or promote the number and growth of ACF in animal models. Although only a small proportion of ACF progress to become colon tumors, the number and multiplicity of these early lesions are predictive of tumor incidence (3). Because ACF occur at an early stage of carcinogenesis, these lesions have been used effectively as a biological endpoint to study the effects of carcinogens and modifying agents (2-5) and the mechanisms involved (5).

Dietary factors have been shown to strongly influence colon cancer risk, and it has been suggested that up to $90 \%$ of colon cancers may be preventable by dietary changes (6). Animal fat can increase cancer incidence, while fruit and vegetable intake can decrease the incidence (7). The diet can contain mutagens that initiate carcinogenesis or promoters that enhance progression. Alternatively, dietary factors may prevent initiation of carcinogenesis by inactivating or removing mutagens, or they may inhibit progression by detoxifying promoters or by directly inhibiting the progression process.

There has been considerable interest in using dietary agents to reduce cancer risk (8). Dithiolethiones and isothiocyanates, which occur in cruciferous vegetables are among the most potent cancer chemopreventive agents known and may exert their protective effects by inducing phase II detoxifying enzymes like NAD(P)H:quinone oxidoreductase 1 (NQO1), glutathione S-transferases (GST) and UDP-glucuronyltrans- 
ferases (UGT) $(9,10)$. These enzymes can inhibit initiation of carcinogenesis by directly modifying and inactivating carcinogens and/or by modifying and increasing their conjugation to glutathione and glucuronic acid leading to their excretion from cells (11). For example, Sugie et al (12) found a lower incidence of colon tumors in animals fed benzyl isothiocyanate prior to carcinogen exposure. However, these agents may also work at the post-initiation stage of carcinogenesis, and may directly induce apoptosis in cancer cells (13). Smith et al (13) found fewer ACF in animals fed allyl isothiocyanate after carcinogen exposure. The dithiolethione, oltipraz, first used as an antiparasitic agent in humans (14), also protects against carcinogenesis in the colon and other sites in rodent models $(15,16)$. In addition, clinical chemoprevention trials with oltipraz showed that it can be safely given to humans and that it reduces activation and increases excretion of the hepatic carcinogen, aflatoxin M1 (17-20). For colon cancer, oltipraz increased the levels of NQO1, GST and UGT in the colons of F344 rats and reduced the number of colon tumors induced by the carcinogen, azoxymethane (AOM), when given either before or after the carcinogen (21). Thus, not all the mechanisms involved in the preventative effects of dithiolethiones and isothiocyanates are known, and it is not clear what role each of the phase II enzymes plays in these mechanisms.

We previously showed that in contrast to the findings in F344 rats, dietary oltipraz selectively induces NQO1 in the colon of Sprague-Dawley rats without increasing the levels of GST or UGT in these animals (22). Using this animal model, we demonstrated that induction of NQO1 by oltipraz prior to administration of carcinogens significantly decreased the formation of ACF in the colons of these rats, but did not alter the distribution of crypt multiplicities. These results provided the first direct evidence that induction of NQO1 alone, without induction of other phase II detoxifying enzymes, can inhibit initiation of colon carcinogenesis, suggesting that this enzyme plays a role in inhibiting carcinogen-induced colon cancer.

NQO1 is a flavoenzyme that catalyzes two-electron reduction of quinones and nitrogen oxides $(23,24)$. A major function of this enzyme may be to decrease formation of reactive oxygen species by decreasing one-electron reductions and the associated redox cycling (25). However, it may also play a role in cancer prevention $(23,26)$ and has been shown to activate some anticancer drugs (27). NQO1 consists of two identical protein subunits of $30 \mathrm{kDa}$ whose expression is transcriptionally controlled (23). The enzyme is induced by a wide variety of inducers (28). The induction pathway may involve a cytosolic redox signal which alters expression and/or interaction of transcriptional factors like Jun, Nrf, Maf, Fos and Fra with the xenobiotic response element and the antioxidant response element (29-35). The enzyme is ubiquitous in eukaryotes but levels vary in different tissues $(23,35,36)$. An inactivating polymorphism of the NQO1 gene involving a change from cytosine to thymine at base 609 occurs at high frequency in the human population, resulting in reduced NQO1 activity in $40 \%$ of the population and no NQO1 activity in $10 \%$ of the population (36-38).

NQO1 may play a role in inhibiting colon cancer at various stages of tumorigenesis. As we have shown (22), it can prevent initiation of colon carcinogenesis likely by detoxifying carcinogens. There is also evidence that NQO1 may inhibit colon carcinogenesis at the post-initiation stage. For example, oltipraz given after initiation of carcinogenesis by AOM induced NQO1 together with GST and UGT, and decreased tumor formation in F344 rats (21). In addition, inducers of phase II detoxifying enzymes have been shown to increase apoptosis in cells in colonic crypts when given after a colon carcinogen (13). Thus, phase II detoxifying enzymes may inhibit colon carcinogenesis at the post-initiation stage; however, it is not clear whether NQO1 alone can have this effect. Studies have shown that NQO1 can stabilize p53 and increase apoptosis in human colon cancer cells (39) and in mouse cells (40). Thus, NQO1 may increase apoptosis of initiated cells in colonic crypts and this could be enhanced by inducers of this enzyme.

In this study we investigated if selective induction of NQO1 in Sprague-Dawley rats by the chemopreventive agent, oltipraz, given after initiation of colon carcinogenesis by AOM inhibited the formation of pre-neoplastic lesions and tumors in the rats. In addition, we examined the effect of postinitiation induction of NQO1 on apoptosis in cells in ACF as a possible mechanism for the inhibition of carcinogenesis.

\section{Materials and methods}

Sprague-Dawley 5-6 week male rats were purchased from the University of Manitoba Central Animal Care Breeding Facility, Winnipeg, Canada and were maintained according to institutional regulations. Modified AIN-76A powdered diet containing $13 \%$ dextrose and $52 \%$ corn starch was obtained from Harlan Teklad (Madison, WI). Oltipraz was obtained from LKT Laboratories (St. Paul, MN). Modified AIN-76A diet containing 200 ppm oltipraz was prepared by Harlan Teklad. AOM and 3-[4,5-dimethythiazol-2-yl]-2,5diphenyltetrazolium bromide were obtained from Sigma (St. Louis, MO). All studies with animals were approved by the Protocol Management and Review Committee of the University of Manitoba and followed the guidelines of the Canadian Council on Animal Care.

For studies of induction of phase II enzymes, SpragueDawley rats were fed control diet or diet containing 200 ppm oltipraz for up to 27 weeks. Rats were euthanized at $6,15,21$ and 27 weeks and colon mucosa were excised. NQO1, GST and UGT activities were measured as previously described (41-43). Enzyme activities in colon mucosa of control fed and inducer fed animals at each time-point were compared by t-tests.

For studies of the effect of oltipraz on AOM-induced ACF, Sprague-Dawley rats were fed control diet from Week -2 to Week 0 (Fig. 1). At Week -1 and Week 0 rats received one treatment with $\mathrm{AOM}$ at $15 \mathrm{mg} / \mathrm{kg}$ by s.c. injection. Starting $24 \mathrm{~h}$ after the second AOM injection, rats were fed control diet or control diet containing 200 ppm oltipraz for 12 weeks. All the rats were then euthanized, colons were removed, fixed in $10 \%$ buffered formalin and stained with methylene blue. Each colon was cut into 3 equal parts representing the proximal, middle and distal sections of the colon, and ACF number and multiplicity in each section and in the whole colon were enumerated as we have described previously $(2,3)$. 


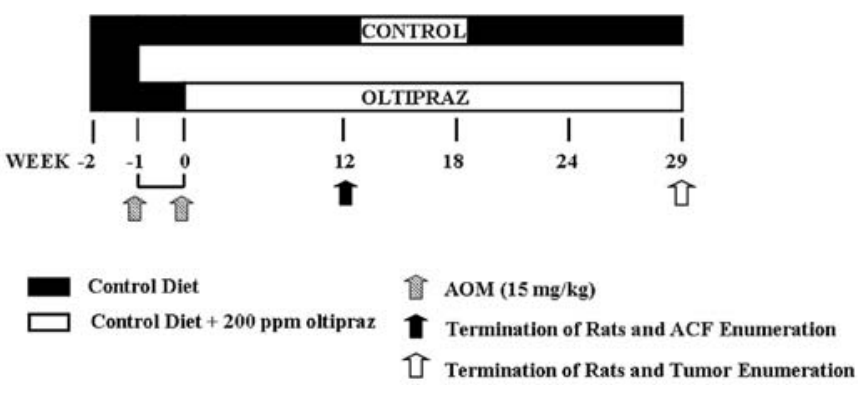

Figure 1. Feeding and treatment schedule for rats.

$\mathrm{ACF}$ numbers in the two treatment groups were compared by one-way analysis of variance (Student-Newman-Keuls Method). The distribution of crypt multiplicities in the two groups was analyzed by repeated measures ANOVA.

To determine the effect of oltipraz on apoptosis in ACF, paraffin blocks were prepared from each of the 3 colon sections obtained from each rat euthanized at 12 weeks following the last AOM injection. Four $(0.5 \mu \mathrm{m})$ transverse sections were cut from each block representing sections of the top, middle and bottom of the colon crypts, and the sections were stained with hematoxylin and eosin. The total number of cells and the number of apoptotic cells in 1 to 8 randomly selected ACF from each block were enumerated and the total number of cells and apoptotic cells in the 4 sections for each ACF were combined. The \% of apoptotic cells in each ACF enumerated was calculated and results obtained for colons from the two treatment groups were compared by Kruskal-Wallis one-way analysis of variance on ranks. The total number of cells and the number of apoptotic cells in randomly selected normal crypts from the same paraffin blocks were also enumerated and the total number of cells and apoptotic cells in the 4 sections for each crypt were combined. The $\%$ of apoptotic cells in each normal crypt enumerated was calculated and results obtained for colons from the two treatment groups were compared by one-way analysis of variance.

For studies of the effect of oltipraz on AOM-induced colon adenomas and tumors, Sprague-Dawley rats were fed control diet from Week -2 to Week 0 (Fig. 1). At Week -1 and Week 0 rats received one treatment with $\mathrm{AOM}$ at $15 \mathrm{mg} / \mathrm{kg}$ by s.c. injection. Starting $24 \mathrm{~h}$ after the second AOM injection rats were fed control diet or control diet containing 200 ppm oltipraz for 29 weeks. All the rats were then euthanized, colons were removed, fixed in $10 \%$ buffered formalin and stained with methylene blue. Each colon was cut into 3 equal parts representing the proximal, middle and distal sections, and adenoma and tumor numbers in each section and the whole colon were enumerated as we have described previously (44). Adenomas were defined as lesions $>2$ and $\leq 10 \mathrm{~mm}^{3}$, while tumors were defined as lesions $>10 \mathrm{~mm}^{3}$. Adenoma and tumor numbers in the two treatment groups were compared by Kruskal-Wallis one-way analysis of variance on ranks.

\section{Results}

Effect of oltipraz on induction of phase II detoxifying enzymes in rat colons. Sprague-Dawley rats were fed control diet or control diet containing 200 ppm oltipraz for up to 27 weeks. At 6, 1521 and 27 weeks rats were euthanized, colon mucosa were excised and NQO1, GST and UGT activities were measured. NQO1 activity at all time-points was increased by 2.1- to 2.6-fold in the colon mucosa of rats fed the oltipraz containing diet compared with rats fed control diet $(\mathrm{p}<0.001)$ (Fig. 2). In contrast, rats fed oltipraz containing diet did not have increased GST or UGT activities in the colon mucosa.
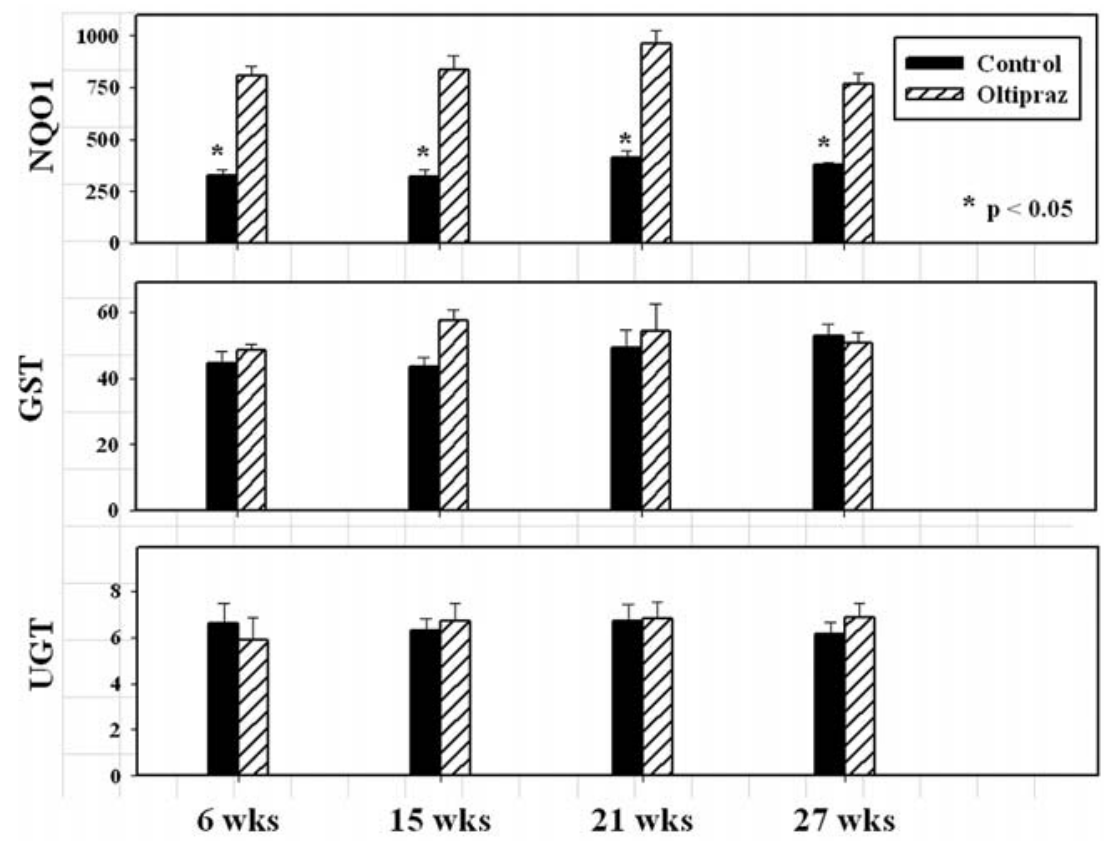

Figure 2. Effect of oltipraz containing diet on phase II detoxifying enzymes in rat colons. Sprague-Dawley rats were fed control diet or diet containing 200 ppm oltipraz for up to 27 weeks. Rats were euthanized at 6, 15, 21 and 27 weeks and colon mucosa were excised. NQO1, GST and UGT activities were measured. Bars represent the mean \pm SE of enzyme activities from 6 to 8 rats. Control fed and oltipraz animals at each time were compared by t-tests. 


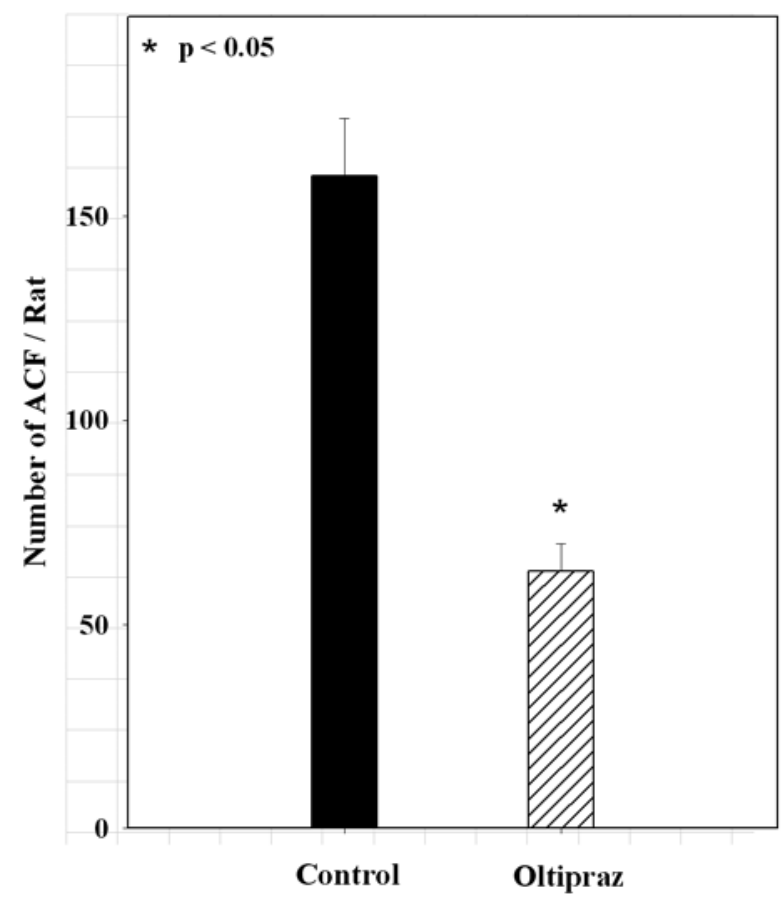

Figure 3. Effect of oltipraz containing diet on ACF formation. SpragueDawley rats were fed control diet from Week -2 to Week 0. At Week -1 and Week 0 rats received one treatment with $\mathrm{AOM}$ at $15 \mathrm{mg} / \mathrm{kg}$ by s.c. injection. Starting $24 \mathrm{~h}$ after the second AOM injection, rats were fed control diet or control diet containing 200 ppm oltipraz for 12 weeks. Rats were then euthanized, colons were removed and ACF numbers were enumerated. Bars represent the mean $\pm \mathrm{SE}$ of the number of $\mathrm{ACF} / \mathrm{rat}$ from 18 rats. $\mathrm{ACF}$ numbers in the two treatment groups were compared by a t-test.

Table I. Effect of post-initiation oltipraz on crypt multiplicity. ${ }^{\mathrm{a}}$

\begin{tabular}{lccccc}
\hline & \multicolumn{5}{c}{ Crypt multiplicity (as \% of total) } \\
\cline { 2 - 6 } Group & 1 & 2 & 3 & 4 & $>4$ \\
\hline Control & 29.2 & 38.1 & 24.9 & 14.3 & 6.1 \\
Oltipraz & 23.1 & 30.9 & 18.7 & 17.9 & 9.4 \\
\hline
\end{tabular}

${ }^{\mathrm{a}} \mathrm{AOM}$ treated rats were fed control diet or control diet containing 200 ppm oltipraz. At 12 weeks after the last AOM treatment the ACF number and multiplicity in each colon were enumerated. The $\%$ of ACF with each multiplicity was calculated for each colon and the means for the two treatment groups are presented.

Effect of post-initiation oltipraz on ACF formation. Sprague-Dawley rats were fed control diet from Week -2 to Week 0 and were treated with $15 \mathrm{mg} / \mathrm{kg} \mathrm{AOM}$ at Week -1 and Week 0 (Fig. 1). Starting $24 \mathrm{~h}$ after the second AOM treatment, the rats were fed control diet or control diet containing $200 \mathrm{ppm}$ oltipraz for 12 weeks. The rats were euthanized, colons were removed and sectioned, and ACF number and multiplicity were enumerated. Rats fed oltipraz containing diet had 66.2 $\pm 7.3 \mathrm{ACF} / \mathrm{rat}(\mathrm{n}=18)$ while rats

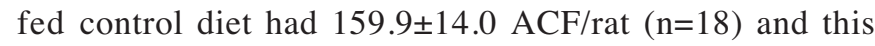
difference was statistically different $(\mathrm{p}<0.001)$ (Fig. 3). The oltipraz diet decreased ACF formation in all of the colon

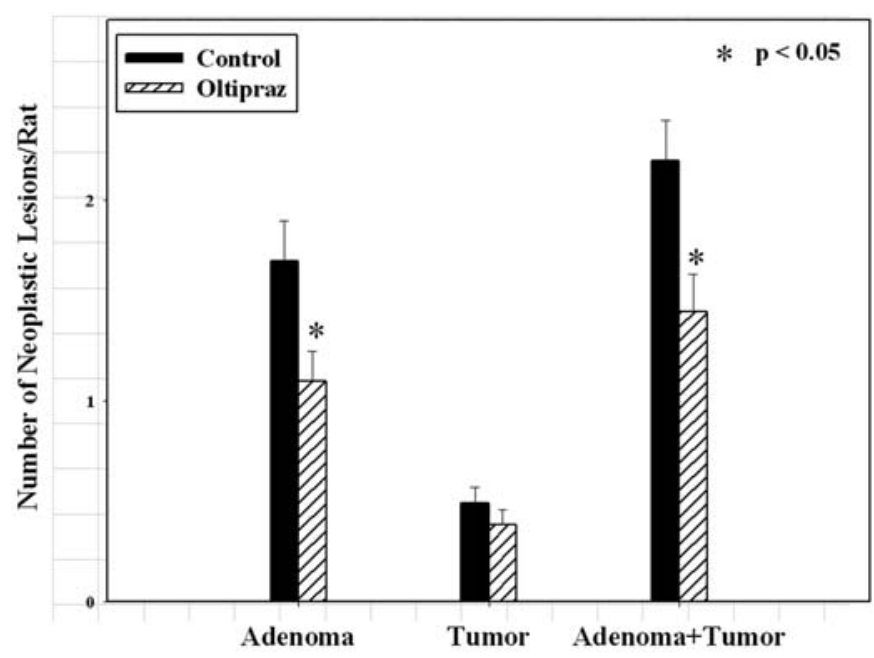

Figure 4. Effect of oltipraz containing diet on adenoma and tumor formation. Sprague-Dawley rats were fed control diet from Week -2 to Week 0. At Week -1 and Week 0 rats received one treatment with AOM at $15 \mathrm{mg} / \mathrm{kg}$ by s.c. injection. Starting $24 \mathrm{~h}$ after the second AOM injection, rats were fed control diet or control diet containing 200 ppm oltipraz for 29 weeks. Rats were then euthanized, colons were removed and adenoma and tumor numbers were enumerated. Bars represent the mean $\pm \mathrm{SE}$ of the number of adenomas, tumors or adenomas + tumors/rat from 69 to 76 rats. Adenoma, tumor and adenoma + tumor numbers in the two treatment groups were compared by one-way analysis of variance.

Table II. Effect of post-initiation oltipraz on tumor incidence. ${ }^{a}$

\begin{tabular}{lccc}
\hline Group & No. of rats & $\begin{array}{c}\text { No. of rats } \\
\text { with tumors }\end{array}$ & $\begin{array}{c}\text { Tumor } \\
\text { incidence (\%) }\end{array}$ \\
\hline Control & 69 & 25 & 36.2 \\
Oltipraz & 76 & 23 & 30.3 \\
\hline
\end{tabular}

${ }^{\mathrm{a} A O M}$-treated rats were fed control diet or control diet containing $200 \mathrm{ppm}$ oltipraz. At 29 weeks after the last AOM treatment the number of rats that had colon tumors was enumerated. The $\%$ of rats with tumors was calculated.

sections. However, oltipraz did not significantly alter the distribution of crypt multiplicities of the ACF (Table I).

Effect of post-initiation oltipraz on adenoma and tumor formation. Sprague-Dawley rats were fed control diet from Week -2 to Week 0 and were treated with $15 \mathrm{mg} / \mathrm{kg} \mathrm{AOM}$ at Week -1 and Week 0 (Fig. 1). The rats were then fed control diet or control diet containing 200 ppm oltipraz for 29 weeks starting $24 \mathrm{~h}$ after the second AOM treatment. The rats were euthanized, colons were removed and adenomas and tumors were enumerated. Rats fed oltipraz containing diet had 1.0 \pm 0.1 adenomas/rat $(n=76)$ while rats fed control diet had $1.7 \pm 0.2$ adenomas/rat $(n=69)$ and this difference was statistically significant $(\mathrm{p}<0.02)$ (Fig. 4). Rats fed oltipraz diet had $0.37 \pm 0.08$ tumors/rat $(n=76)$ while rats fed control diet had $0.49 \pm 0.08$ tumors/rat $(n=69)$ but this difference was not statistically significant $(\mathrm{p}<0.29)$. However, when adenomas and tumors in the two groups of rats were compared the oltipraz fed rats 
Table III. Effect of post-initiation oltipraz on apoptosis in ACF. ${ }^{a}$

\begin{tabular}{lccc}
\hline Group & $\begin{array}{c}\text { No. of ACF } \\
\text { analyzed }\end{array}$ & $\begin{array}{c}\text { \% Apoptotic cells } \\
\text { in ACF (mean } \pm \text { SE) }\end{array}$ & p-value \\
\hline Control & $303^{\text {b }}$ & $0.72 \pm 0.04$ & \\
Oltipraz & $293^{\text {b }}$ & $1.16 \pm 0.06$ & $<0.001$ \\
\hline
\end{tabular}

${ }^{a} \mathrm{AOM}$-treated rats were fed control diet or control diet containing 200 ppm oltipraz. At 12 weeks after the last AOM treatment paraffin blocks were prepared from the colons and 4 transverse sections were cut from each block. The total number of cells and the number of apoptotic cells in randomly selected ACF were enumerated and the $\%$ of apoptotic cells in each ACF enumerated was calculated. ${ }^{b}$ From 18 rats.

Table IV. Effect of post-initiation oltipraz on apoptosis in normal colonic crypts. ${ }^{\text {a }}$

\begin{tabular}{cccc}
\hline Group & $\begin{array}{c}\text { No. of normal } \\
\text { crypts analyzed }\end{array}$ & \% Apoptotic \\
& $\begin{array}{c}\text { cells in normal crypts } \\
(\text { mean } \pm \text { SE) }\end{array}$ &
\end{tabular}

\begin{tabular}{llll}
\hline Control & $22^{\mathrm{b}}$ & $2.69 \pm 0.57$ & \\
Oltipraz & $23^{\mathrm{b}}$ & $3.29 \pm 0.47$ & $\mathrm{NS}^{\mathrm{c}}$ \\
\hline
\end{tabular}

${ }^{a} \mathrm{AOM}$-treated rats were fed control diet or control diet containing 200 ppm oltipraz. At 12 weeks after the last AOM treatment paraffin blocks were prepared from the colons and 4 transverse sections were cut from each block. The total number of cells and the number of apoptotic cells in randomly selected normal crypts were enumerated and the $\%$ of apoptotic cells in each normal crypt enumerated was calculated. ${ }^{b}$ From 6 rats; ${ }^{c} \mathrm{NS}$, not significant.

had significantly fewer neoplastic lesions $(\mathrm{p}<0.02)$. This effect was similar in all sections of the colon. In addition, the tumor incidence rate (\% of rats with tumors) was $30.3 \%$ in rats that were fed oltipraz containing diet while rats that were fed control diet had a tumor incidence of $36.2 \%$ (Table II).

Effect of post-initiation oltipraz on apoptosis in ACF. SpragueDawley rats were fed control diet from Week -2 to Week 0 and were treated with $15 \mathrm{mg} / \mathrm{kg} \mathrm{AOM}$ at Week -1 and Week 0 (Fig. 1). The rats were then fed control diet or control diet containing $200 \mathrm{ppm}$ oltipraz for 12 weeks starting $24 \mathrm{~h}$ after the second AOM treatment. The rats were euthanized, and the colons were removed and sectioned. Paraffin blocks were prepared from the colons and 4 transverse sections were cut from each block. The total number of cells and the number of apoptotic cells in randomly selected ACF were enumerated and the $\%$ of apoptotic cells in each ACF enumerated was calculated (Table III). The \% of apoptotic cells in ACF was $1.16 \pm 0.06$ ( $n=293$ ACF from 18 rats) from oltipraz fed rats and $0.72 \pm 0.04$ ( $n=303$ ACF from 18 rats) from control fed rats and this difference was statistically significant $(\mathrm{p}<0.001)$.
Using the same paraffin sections the total number of cells and the number of apoptotic cells in randomly selected normal colonic crypts were enumerated and the \% of apoptotic cells in each crypt enumerated was calculated (Table IV). The $\%$ of apoptotic cells in normal crypts was $3.29 \pm 0.47$ $(\mathrm{n}=23$ normal crypts from 6 rats) from oltipraz fed rats and $2.69 \pm 0.57$ ( $\mathrm{n}=22$ normal crypts from 6 rats) from control fed rats and these results were not statistically different.

\section{Discussion}

There is considerable evidence that phase II detoxifying enzymes play a significant role in preventing carcinogen induced colon cancer, and inducers of these enzymes have been extensively investigated as chemopreventive agents $(11-16,21,45,46)$. Despite this effort the contribution of NQO1 to this effect remains unclear. We have demonstrated that selective induction of NQO1 in rat colon, without inducing GST or UGT, prior to treatment with a carcinogen significantly inhibited the formation of ACF (22). This finding suggests that NQO1 plays a role in preventing initiation of colon carcinogenesis. However, evidence suggests that induction of phase II detoxifying enzymes may also inhibit colon carcinogenesis at the post-initiation stage $(13,21)$. In this study we used the AOM induced colon cancer University of Manitoba Sprague-Dawley rat model to determine if NQO1 alone can contribute to prevention of colon carcinogenesis at the post-initiation stage. We have previously shown that feeding these rats a diet containing the chemopreventive agent, oltipraz, selectively induces NQO1 in the colons without inducing GST or UGT (22). Thus, we examined whether post-initiation induction of NQO1 altered AOM induced $\mathrm{ACF}$, adenoma and tumor formation in these rats.

Oltipraz (200 ppm) added to the diet of the SpragueDawley rats increased the level of NQO1 activity in the colons of these rats by more than 2-fold over a period of more than 27 weeks, but did not significantly increase the levels of GST or UGT activities (Fig. 2). Rats fed oltipraz containing diet starting one day after treatment with the colon carcinogen, AOM, had almost $60 \%$ fewer ACF after 12 weeks compared with rats fed a control diet $(\mathrm{p}<0.001)$ (Fig. 3). Similarly, rats fed oltipraz containing diet after AOM treatment developed $40 \%$ fewer colon adenomas after 29 weeks than rats fed a control diet $(\mathrm{p}<0.01)$ (Fig. 4). There were also fewer colon tumors after 29 weeks in the oltipraz fed rats compared with control fed rats, but this difference did not reach statistical significance. However, the combination of adenomas and tumors was significantly lower in oltipraz fed rats than in control fed rats $(\mathrm{p}<0.02)$. In addition, the tumor incidence ( $\%$ of rats developing tumors) was lower in the oltipraz fed animals. Together, these results suggest that NQO1 contributes to inhibition of colon carcinogenesis at the post-initiation stage. However, because oltipraz can induce genes other than NQO1, GST and UGT, we can not rule out a role for some of these gene products in the effects we observed.

There may be several explanations for the lack of a significant difference in tumor formation in the oltipraz fed rats compared with the control fed rats. The most likely is the low number of tumors obtained in this study compared with 
previous studies using this rat model. The tumor incidence and tumor frequency (tumors/rat) were $36.2 \%$ and $0.49 \%$, respectively, for the control group in this study compared with $71.4 \%$ and $1.54 \%$, respectively, in a previous study from our laboratory using the same experimental design (5). While the reasons for the lower number of tumors are not known, the lower numbers of tumors along with the normal experimental variation from rat to rat likely accounted for the lack of statistical significance between the two experimental groups. It may have been possible to increase the tumor frequency in this study by increasing the time after AOM treatment for enumeration of the tumors, but this was not technically feasible because of the development of tumors in the ears of some of the rats after 29 weeks. University regulations required that these animals be euthanized as soon as these tumors appeared. Although the current study did not demonstrate statistically significant inhibition of tumor formation in oltipraz fed rats compared with control fed rats, we did observe a significant decrease in the number of neoplastic lesions (adenomas + tumors) in the oltipraz fed animals.

The oltipraz diet produced similar effects on ACF, adenoma and tumor formation in all sections of the colon. This finding suggests that oltipraz induced NQO1 in all parts of the colon, and that NQO1 had a similar effect on the carcinogenesis process in all parts of the colon. In addition, the finding that oltipraz did not affect the ACF crypt multiplicity distribution suggests that NQO1 may be producing its effect at an early stage of the carcinogenesis process. A possible mechanism for this effect may be that the induction of NQO1 by oltipraz may increase apoptosis in colonic epithelial cells initiated by the colon carcinogen, AOM. Inducers of phase II detoxifying enzymes have been shown to increase apoptosis in cells in colonic crypts when given after a colon carcinogen (13). Studies have also shown that NQO1 can stabilize p53 and increase apoptosis in human colon cancer cells (39) and in mouse cells (40). Thus, induction of NQO1 by oltipraz may stabilize p53 in the initiated cells resulting in increased apoptosis and reduced formation of ACF.

To test this hypothesis we determined the effect of postinitiation oltipraz on apoptosis in cells in ACF. We found that there was a $60 \%$ increase in the percentage of apoptotic cells in ACF from oltipraz fed rats compared with ACF from control fed rats. This finding provides support for the hypothesis that NQO1 inhibits colon carcinogenesis at an early post-initiation stage by increasing apoptosis of initiated cells preventing them from progressing to a neoplastic state.

We also determined the percentage of apoptotic cells in normal colonic crypts from oltipraz fed rats and control fed rats. The percentage of apoptotic cells in normal crypts was 4-fold greater than in ACF from control fed rats, but the oltipraz diet did not increase apoptosis in the normal crypts. Our finding of a lower percentage of apoptosis in ACF compared with normal crypts is similar to results we observed previously (47), and may reflect an enhanced survival mechanism in the initiated cells of ACF that contributes to their increased proliferation and tumor formation.

In summary, this study demonstrated that post-initiation induction of NQO1 decreases the formation of carcinogen induced pre-neoplastic and neoplastic colonic lesions. This may be due to increased apoptosis in initiated colon epithelial cells due to stabilization of p53 protein by NQO1 in these cells. Thus, NQO1 may play a role in preventing colon carcinogenesis at the post-initiation stage, and this enzyme may be an important target for chemoprevention of colon cancer. This conclusion is supported by our finding from a case control study (48) that an inactivating NQO1 polymorphism may be a risk factor for development of colon cancer in humans.

\section{Acknowledgements}

The study was supported by grants from the Canadian Institutes of Health Research and CancerCare Manitoba Foundation.

\section{References}

1. Cancer Facts and Figures 2003. American Cancer Society Inc, 2003.

2. Bird RP: Aberrant crypt foci system to study cancer preventive agents in the colon. In: Methods in Molecular Medicine. Vol 14 Tumor marker protocols. Hanausek M and Walaszek Z (eds). Humana Press, Totowa, pp465-474, 1998.

3. Bird RP: Role of aberrant crypt foci in understanding the pathogenesis of colon cancer. Cancer Lett 93: 55-71, 1995.

4. Magnuson BA, Carr I and Bird RP: Ability of aberrant crypt foci characteristics to predict colonic tumour incidence in rats fed cholic acid. Cancer Res 53: 4499-4504, 1993.

5. Bird RP, Yao K, Lasko CM and Good CK: Inability of lowor high-fat diet to modulate late stages of colon carcinogenesis in Sprague Dawley rats. Cancer Res 56: 2896-2899, 1996.

6. Doll R and Peto R: The causes of cancer: quantitative estimates of avoidable risks of cancer in the United States today. J Natl Cancer Inst 66: 1191-1308, 1981.

7. Kim Y and Mason JB: Nutrition chemoprevention of gastrointestinal cancers: a critical review. Nutr Rev 54: 259-279, 1996.

8. Wargovich MJ, Chen C-D, Jimenez A, et al: Aberrant crypts as a biomarker for colon cancer: evaluation of potential chemopreventive agents in the rat. Cancer Epidemiol Biomarkers Prev 5: 355-360, 1996.

9. Morse MA and Stoner GD: Cancer chemoprevention. Carcinogenesis 14: 1737-1746, 1993 .

10. Hecht SS: Chemoprevention by isothiocyanates. J Cell Biochem 22 (Suppl): 195-209, 1995.

11. Wattenberg LW: An overview of chemoprevention: current status and future prospects. Proc Soc Exp Biol Med 216: 133-141, 1997.

12. Sugie S, Okamoto K, Okumura A, Tanaka T and Mori H: Inhibitory effects of benzyl thiocyanate and benzyl isothiocyanate on methylazoxymethanol acetate-induced intestinal carcinogenesis in rats. Carcinogenesis 15: 1555-1560, 1994.

13. Smith T, Musk SRR and Johnson IT: Allyl isothiocyanate selectively kills undifferentiated HT29 cells in vitro and suppresses aberrant crypt foci in the colonic mucosa of rats. Biochem Soc Trans 24: 381S, 1996.

14. Archer S: The chemotherapy of schistosomiasis. Ann Rev Pharmacol Toxicol 25: 485-508, 1985.

15. Wattenberg LW and Bueding E: Inhibitory effects of 5-(2pyrazinyl)-4-methyl-1,2-dithiol-3-thione (Oltipraz) on carcinogenesis induced by benzo[a]pyrene, diethylnitrosamine and uracil mustard. Carcinogenesis 7: 1379-1381, 1986.

16. Kensler TW, Groopman JD and Roebuck BD: Chemoprotection by oltipraz and other dithiolethiones. In: Cancer Chemoprevention. Wattenberg L, Lipkin M, Boone C and Kelloff G (eds). CRC Press, Boca Raton, pp205-225, 1992.

17. Zhang B-C, Zhu Y-R, Wang J-B, et al: Oltipraz chemoprevention trial in Qidong, Jiangsu Province, People's Republic of China. J Cell Biochem (Suppl) 28/29: 166-173, 1997.

18. Kensler TW, He X, Otieno, M, et al: Oltipraz chemoprevention trial in Qidong, People's Republic of China: modulation of serum aflatoxin albumin adduct biomarkers. Cancer Epidemiol Biomarkers Prev 7: 127-134, 1998. 
19. Wang JS, Shen X, He X, et al: Protective alterations in phase 1 and 2 metabolism of aflatoxin B1 by oltipraz in residents of Qidong, People's Republic of China. J Natl Cancer Inst 91: 347-354, 1999.

20. Camoirano A, Bagnasco M, Bennicelli C, et al: Oltipraz chemoprevention trial in Qidong, People's Republic of China: results of urine genotoxicity assays as related to smoking habits. Cancer Epidemiol Biomarkers Prev 10: 775-783, 2001.

21. Rao CV, Rivenson A, Katiwalla M, Kelloff GJ and Reddy BS: Chemopreventive effect of Oltipraz during different stages of experimental colon carcinogenesis induced by azoxymethane in male F344 rats. Cancer Res 53: 2502-2506, 1993.

22. Begleiter A, Sivananthan K, Curphey TJ and Bird RP: Induction of NAD(P)H quinone:oxidoreductase inhibits carcinogen induced aberrant crypt foci in colons of Sprague-Dawley rats. Cancer Epidemiol Biomarkers Prev 12: 566-572, 2003.

23. Ross D, Siegel D, Beall H, Prakash AS, Mulcahy RT and Gibson NW: DT-diaphorase in activation and detoxification of quinones. Cancer Metast Rev 12: 83-101, 1993.

24. Riley RJ and Workman P: DT-diaphorase and cancer chemotherapy. Biochem Pharmacol 43: 1657-1669, 1992.

25. Ernster L: DT-diaphorase: a historical review. Chem Scr 27A: $1-13,1987$

26. Begleiter A, Leith MK, Curphey TJ and Doherty GP: Induction of DT-diaphorase in cancer chemoprevention and chemotherapy. Oncol Res 9: 371-382, 1997.

27. Begleiter A, Robotham E, Lacey G and Leith MK: Increased sensitivity of quinone resistant cells to mitomycin C. Cancer Lett 45: 173-176, 1989.

28. Prestera T, Zhang Y, Spencer SR, Wilczak CA and Talalay P: The electrophile counterattack response: protection against neoplasia and toxicity. Adv Enzyme Regul 33: 281-296, 1993.

29. Yao KS, Xanthoudakis S, Curran T and O'Dwyer PJ: Activation of AP-1 and of a nuclear redox factor, Ref-1, in the response of HT29 colon cancer cells to hypoxia. Mol Cell Biol 14: 5997-6003, 1996.

30. Venugopal R and Jaiswal AK: Nrf1 and Nrf2 positively and cFos and Fral negatively regulate the human antioxidant response element-mediated expression of NAD(P)H:quinone oxidoreductase1 gene. Proc Natl Acad Sci USA 93: 14960-14965, 1996.

31. Venugopal R and Jaiswal AK: Nrf2 and Nrf1 in association with Jun proteins regulate antioxidant response element-mediated expression and coordinated induction of genes encoding detoxifying enzymes. Oncogene 17: 3145-3156, 1998.

32. Kepa JK and Ross D: DT-diaphorase activity in NSCLC and SCLC cell lines: a role for fos/jun regulation. Br J Cancer 79: 1679-1684, 1999.

33. Nguyen T, Huang HC and Pickett CB: Transcriptional regulation of the antioxidant response element. J Biol Chem 275: 15466-15467, 2000

34. Favreau LV and Pickett CB: Transcriptional regulation of the rat $\mathrm{NAD}(\mathrm{P}) \mathrm{H}$ :quinone reductase gene. characterization of a DNA-protein interaction at the antioxidant responsive element and induction by 12-o-tetradecanoylphorbol 13-acetate. J Biol Chem 268: 19875-19881, 1993
35. Belinsky $\mathrm{M}$ and Jaiswal AK: NAD(P)H:quinone oxidoreductase 1 (DT-diaphorase) expression in normal and tumor tissues. Cancer Metastasis Rev 12: 103-117, 1993.

36. Schlager JJ and Powis G: Cytosolic NAD(P)H: (quinoneacceptor) oxidoreductase in human normal and tumor tissue: effects of cigarette smoking and alcohol. Int J Cancer 45: 403-409, 1990.

37. Traver RD, Siegel D, Beall HD, Phillips RM, Gibson NW, Franklin WA and Ross D: Characterization of a polymorphism in NAD(P)H:Quinone oxidoreductase (DT-diaphorase). Br J Cancer 75: 69-75, 1997.

38. Kelsey KT, Ross D, Traver RD, et al: Ethnic variation in the prevalence of a common $\mathrm{NAD}(\mathrm{P}) \mathrm{H}$ quinone oxidoreductase polymorphism and its implications for anti-cancer chemotherapy. Br J Cancer 76: 852-854, 1997.

39. Asher G, Lotem J, Cohen B, Sachs L and Shaul Y: Regulation of p53 stability and p53-dependent apoptosis by NADH quinone oxidoreductase 1. Proc Natl Acad Sci USA 98: 1188-1193, 2001.

40. Long DJ II, Gaikwad A, Multani A, Pathak S, Montgomery CA, Gonzalez FJ and Jaiswal AK: Disruption of the NAD $(\mathrm{P}) \mathrm{H}$ : quinone oxidoreductase 1 (NQO1) gene in mice causes myelogenous hyperplasia. Cancer Res 62: 3030-3036, 2002.

41. Doherty GP, Leith MK, Wang X, Curphey TJ and Begleiter A: Induction of DT-diaphorase by 1,2-dithiole-3-thiones in human tumor and normal cells and effect on antitumor activity of bioreductive agents. Br J Cancer 77: 1241-1252, 1998.

42. Habig WH, Pabst MJ and Jakoby WB: Glutathione S-transferase. The first enzymatic step in mercapturic acid formation. J Biol Chem 249: 7130-7139, 1974.

43. Chengelis CP: Age- and sex-related changes in epoxide hydrolase, UDP-glucuronosyl transferase, gluathione Stransferase, and PAPS sulfotransferase in Sprague-Dawley rats. Xenobiotica 18: 1225-1237, 1988.

44. Good CK, Lasko CM, Adam J and Bird RP: Diverse effect of fish oil on the growth of aberrant crypt foci and tumor multiplicity in F344 rats. Nutr Cancer 31: 204-211, 1998.

45. Grubben MJ, Nagengast FM, Katan MB and Peters WH: The glutathione biotransformation system and colorectal cancer risk in humans. Scand J Gastroenterol (Suppl) 234: 68-76, 2001.

46. Wilkinson $\mathrm{J} 4$ th and Clapper ML: Detoxication enzymes and chemoprevention. Proc Soc Exp Biol Med 216: 192-200, 1997.

47. Magnuson BA, Shirtliff N and Bird RP: Resistance of aberrant crypt foci to apoptosis induced by azoxymethane in rats chronically fed cholic acid. Carcinogenesis 15: 1459-1462, 1994.

48. Begleiter A, Hewitt D, Maksymiuk AW, Ross DA and Bird RP: An NQO1 polymorphism is a risk factor for human colon cancer. Cancer Epidemiol Biomarkers Prev 15: 2422-2426, 2006. 